\title{
Research on the Population Migration Trend Algorithm based on Artificial Fish Swarm Algorithm
}

\author{
Xi-Wen BI, Meng XU* \\ College of Information Technology and Media,Beihua University, Jilin 132013, China.
}

\begin{abstract}
KEYWORD: Population Migration Trend Algorithm; Artificial Fish Swarm Algorithm; Convergence
\end{abstract}
\begin{abstract}
Population migration trend is a new kind of evolutionary algorithm proposed recently, which simulates the principle of Population Migration. In this paper, the new search of mechanism is proposed to predict the population migration trend for the visual effect to the convergence of the ASFA(Artificial Fish Swarm Algorithm). the analysis of experiments is obvious that when searching area contracts, the convergence is improved greatly. The numerical experiments show that the mean iteration generation and the least successfully iteration generation of he randomness of population's movement in PMA is less than that of ASFA. And the convergence algorithm shows better local search ability and convergence stability.
\end{abstract}

\section{GENERAL INSTRUCTIONS}

Optimization aim is to find the optimal answer to solve the problem. Optimization in the modern sense of computer technology is essentially the same mathematical theory of comprehensive planning for a problem is concerned, it contains a number of limiting factors (such as strength, cost, technology, technology, etc.), the optimization process is to optimize the use of theory and methods, through an iterative computer calculation to find the optimum design parameter values at the limiting factor, so that the problem in one way or certain aspects of the optimal.

Competition is fierce in today's society, competition between countries has been reflected not only in the military field, but more commonly is penetrated into people's daily life in the economic competition, other man-made factors aside, which is essentially the quality of products and competition between the price by reducing the cost of products, conserve resources, so that the overall performance of the product to achieve optimal, the product more competitive, these requirements can be achieved through the use of optimized design. It can be said today for the most optimized design requirements are more important and urgent than ever before.

AFSA (Artificial Fish Swarm Algorithm, AFS is a sentence based on simulated evolutionary algorithm River fish behavior in basic AFSA, primarily the use of fish feeding, clusters and rearend behavior, from construction the underlying behavior of a single fish to start, through local optimization of each individual fish, the global optimum value in the population to the fore purposes. the algorithm has good ability to overcome local optima achieved global extremes. and algorithm uses only function values of the objective function, the gradient values and other information without special objective function, the search space has a certain adaptive able to. algorithm is not sensitive to the initial value, the choice of the parameters is also not clear limits. also AFSA top-down design ideas, from individual behavior of artificial fish starting to reach the final result of emergence, to optimize the solution to the problem provides a new way to carry out related research is necessary. AFSA is a new way of thinking, from the general to the specific embodiments algorithm design concepts are different from the traditional design and solutions, but it can blend with traditional methods, therefore, whether from specific mathematical problems or to higher levels of management scheduling problem, have a good prospect.

Population migration algorithm and conventional analog evolutionary algorithms, past groups of various algorithms often have some optimization features by simulating natural areas to build the model. The population migration algorithm simulation is the social field of population with the economic center of gravity shift, with increasing population pressure diffusion mechanism that simulates "downwards, with the flow of people to the rich," the law, that is, He said that when the relative overpopulation of a preferential area, population pressure increases, people will move out of the area to find better deals more suited to their preferential areas. This algorithm is designed for the people to provide a new way of thinking and direction. Numerical experiments show that now has a population migration algorithm has good global search capability, but the theoretical basis remains to be expanded. 
Due to a variety of simulated evolutionary algorithm theoretical basis of imperfect people make when using the algorithm can only use their experience and ask questions to understand to select various optimization parameters. Theoretical Basis simulated evolutionary algorithm focused on the exploration of the algorithm search mechanism, convergence, convergence speed, complexity, effectiveness, can solve other issues, and its purpose is to clarify the algorithm works with the shape theory, which simulation algorithm technology for the development and evolution of applications compared with the theoretical basis.

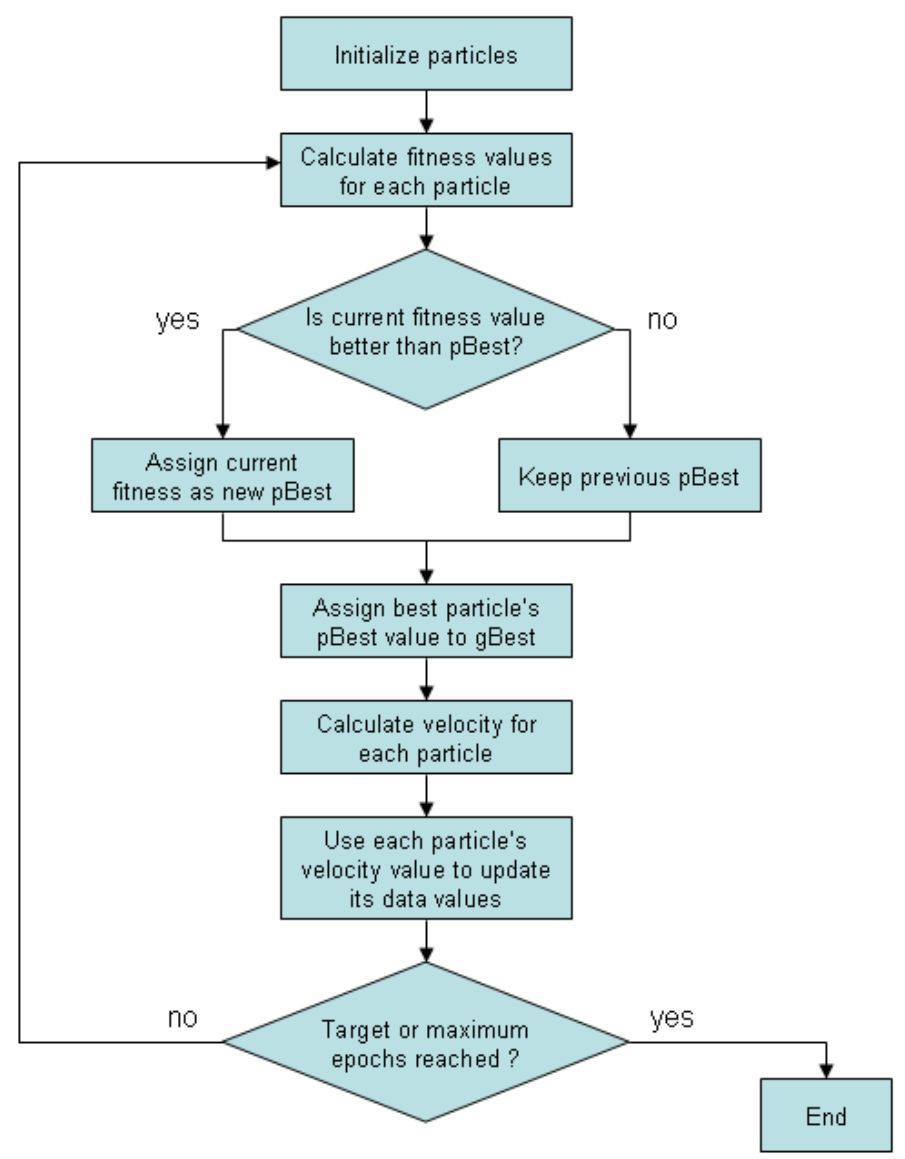

Figure 1. AFSA process

\section{AFSA}

In nature, as long evolution and adaptation, some of the lower forms of life and foraging lifestyle and higher organisms formed their unique different ways, the most obvious difference is that they do not have the advanced intelligent human thought, Because they (such as ants, birds, fish, etc.) are usually gregarious way of life and feeding, so artificial life researchers call this phenomenon as swarm intelligence (SwarmIntelligence), that is, the cluster is composed by an agent collection of their members by direct or indirect (and their local environment action) approach each other, and work together to solve a complex problem of distributed, such as ant colony algorithm, particle swarm optimization algorithm is relatively good application. AFSA is a simulation of natural biological characteristics of intelligent algorithm, it is Li Xiaolei [Zhejiang University and other people since 2002, was first proposed, and has been widely attention of domestic and foreign scholars, the study of the application of the algorithm has been infiltrated the number of applications by solving one-dimensional static optimization problem to solve multi-dimensional development of dynamic combinatorial optimization problems. Currently, the AFSA has become a very active interdisciplinary frontier of research questions.

Through extensive research we found that AFSA has the following characteristics:

(1) only needs the objective function value comparison, is not sensitive to the nature of the objective function;

(2) the algorithm is not sensitive to the initial value, the initial value randomly generated or can be set to a fixed value; 
No (3) algorithms for parameter setting clear limits, a larger allowable range;

(4) the ability of parallel processing algorithms, optimization faster;

(5) algorithm with global optimization capability to quickly jump out of local minima;

AFSA is currently widely used in these areas, based on the algorithm itself features: Reactive power system optimization, optimal power flow calculation unit combinatorial optimization, protection, short-term load forecasting; parameter identification, definite parameters, parameter estimation the system online identification; optimal allocation of resources; VRP scheduling; RBF neural network training; solve combinatorial optimization problems; software reliability optimization design; real-time search engine optimization, feedforward neural network optimization.

From the current research on AFSA point of view, most of the focus on how to apply the AFSA to solve practical problems, while research and optimization algorithm itself, not much to see. Through in-depth study and practice found that although the AFSA has many good features, but there are still some problems itself, such as: the ability to obtain exact solutions is not enough, the system can only obtain satisfactory solution domain; optimization of the area than the large, flat area or in the change, the rate of convergence to global optimal solution slow search efficiency degradation; optimization algorithm generally has a faster initial convergence, convergence is slow and late. These problems exist in the algorithm itself, to a certain extent, also influenced the practical application of the algorithm.

\section{POPULATION MIGRATION ALGORITHM}

Population Migration Algorithm (Population Migration Algorithm, PMA) is a global Chinese scholars Zhou Yonghua, Mao Zongyuan proposed in 2003 a class migration mechanism simulation optimization algorithm. The algorithm is based on the basis of a simple analog mobile population overall understanding of the law on population migration. Population migration algorithm is simulated evolutionary algorithm is an algorithm optimization theory, optimization theory and algorithms is an important branch of mathematics. Population migration algorithm development and have a wide range of applications in mathematics, computer science and engineering fields. Problems algorithm research is discussed in a number of scenarios of what the best solution and how to find the optimal solution. In order to solve a variety of optimization problems, people have developed a variety of optimization algorithms such as simplex method, gradient method, neural network algorithm. These optimization algorithms each has advantages, each have their own scope, but also each have their own limitations. For complex, difficult global objective function optimization problem with some AI problems, these non-traditional optimization methods or applications, or may be applied but the efficiency is not high. In this case, the migration algorithm emerged in the 1960s, and has begun to attract people's attention from the 1980s and is widely used. Algorithm animate. Mode, using a bottom-up design approach, so focus first on construction of artificial fish model. Here the main use of object-oriented technology, and pseudo-code in the form of the $\mathrm{C}++$ language to illustrate.

Population migration algorithm is a simulation of natural biological evolution process and mechanism for solving optimization problems and the search for a class of self-organizing, adaptive artificial intelligence technology. Population migration algorithm has general, parallel, stable, and simple, but also has a strong global search capability. Now has experimental and theoretical analysis shows that when global optimization objective function does not parse the expression, or, though analytic expression but not exactly the valuation, or target function has very many peaks or multi-objective or non-objective function continuous, non-differentiable, highly nonlinear, the analog evolutionary algorithm is very effective. Common analog evolutionary algorithm Holland proposed genetic algorithm (Genetic Algorithms, short GA) immune algorithm, T.Fukuda et al proposed (lmmune Algorithm, called IA), and Rechenberg and Schwefel evolution strategies (Evolution Strategy, referred to as the ES )Wait.

\section{IMPROVED AFSA ITS CONVERGENCE ANALYSIS}

In the evolution of animals, after a long survival of the fittest in nature, forming all kinds of food and way of life, these methods for mankind to solve the problem brought a lot of inspiration and 
encouragement. Animals generally do not have the advanced intelligent human beings have complex logical reasoning ability and comprehensive judgment, their purpose is the simple act of an individual or group to achieve or come to the fore. Animal behavior has the following characteristics:

(1) Adaptability: animal senses to perceive the outside world by a bad environment, and stress to make a variety of responses,

Thus affecting the environment, showing the ability to interact with the environment;

(2) autonomy: some animals have their specific behavior, at different times and in different environments can customize select a certain behavior, without the need for external control or guidance;

(3) blindness: Unlike traditional knowledge-based intelligent systems, has a clear goal, the behavior of a single individual of AFSA is independent, and often between the overall objective is not directly related;

(4) Emergence: The overall objective is to complete the projection during the movement emerged in individual behavior to;

(5) Parallelism: each individual behavior in real time, performed in parallel;

AFSA (AFS aid is based on fish activity characteristic A proposed based on animal behavior municipality optimization mode.

Algorithm animate. Mode, using a bottom-up design approach, so focus first on construction of artificial fish model. Here the main use of object-oriented technology, and pseudo-code in the form of the $\mathrm{C}++$ language to illustrate.

Typically, artificial fish model can be described by the following classes.

Class Artificial Fish\{

inline void autoheal (ENTITY_LIST * \& ENTLIST_List, BODY * \& BODY_HealedBody) \{

if $(($ ENTLIST_List-> count ()$==0))$

\{

$::$ MessageBox (NULL, "entity_list-> count ()$==0$ !", "Message box", MB_OK + MB_ICONINFORMATION);

exit (0);

\}

int iNumFaces $=0$;

int iCount = ENTLIST_List- $>$ count ();

typedef FACE * PFace;

PFace * FACE_FaceArray $=$ new PFace [iCount * 10]; // here to ensure that the space must be allocated sufficient

\section{CONCLUSION}

In this paper, artificial fish population and migration algorithm, population migration algorithm, an improved artificial intelligence optimization algorithm based on a school of fish. The iterative algorithm to find the optimal design parameters under constraints, so to solve the problem of optimal in one aspect or some aspects. And prove the convergence probability artificial fish population migration algorithm based on sexual mathematically. Specific content:

(1) The basic AFSA carried out the corresponding studies and analyzes the influence of various parameters of the algorithm. Detailed analysis of the impact of vision and steps of the algorithm.

(2) Since the AFSA is large or changes in the flat areas, global convergence is slow in its optimization area, low search efficiency. On this point the basic AFSA improved search mechanism.

(3) the improved AFSA algorithm is compared with the basic fish. For improved AFSA numerical experiments, the search area through the analysis of numerical experiments "zoom out" after convergence speed, and the success rate of convergence to the thresholds have been increased. Repeatedly proved average number of iterations of the algorithm and the minimum successful convergence of algebraic improved significantly reduced, and the improved algorithm has better convergence of local refinement capacity and stability. 
(4) analysis of population migration algorithm AFSA and similarities, to find a combination of both based on the similarities between the two points so that the AFSA in swarm intelligence is introduced into the population migration algorithm to construct the population migration algorithm based on artificial fish.

(5) describes the basic structure of hybrid algorithm, mathematically prove the feasibility of the algorithm, and analyzes the hybrid algorithm convergence. Finally, numerical experiments show that the hybrid algorithm has better global convergence and stability than pure or artificial fish population migration algorithm.

\section{Corresponding Author: Meng Xu \\ Acknowledgements:}

1. Vehicle vibration damping characteristics [104900150]

2. professional applications "C Programming Language" case-Teaching Practice [XJQN2016035]

3. Fish Model Based on Artificial Life: Jilin Province Department of Education 2015[137]

\section{REFERENCES}

MY, Jiang; DF, Yuan.Artificial Fish Swarm Algorithm and its applications Dynamics of continuous discrete and impulsive system-series b-applicition \& algorithm, 2007:. 1782-1787

Zhang Libiao, Zhou Chunguang, Liu Xiaohua, etc. for solving constrained optimization evolutionary algorithm, a new issue of Journal of Jilin University (Science Edition), 2004, 42 (4): $85-88$

An algorithm lindane, LI Min-qiang, KOU Jisong Based on Genetic Algorithm for Constrained Optimization of Software, 2003, 12 (4): 628-632

Wang Ling with intelligent optimization algorithm and its optimization technology. Beijing: Tsinghua University Press, 2001.10: 23-25

Liu Jian, Lin Yue, WANG Shu-yun improved particle swarm algorithm and its application of Jilin University (Science Edition) in solving constrained optimization problems, 2005, 4: 84-88

ZHOU Yu, ZHOU Ji-Hong, Wang Yong, a non-parametric optimization evolutionary algorithm punitive function Computer Engineering, 2005, 31 (10): 40-42

Wu Ying. Convergence of Population Migration Algorithm Xi'an science and technology human science, applied mathematics Master ten papers, 2005

Chen Huafeng, Tan Jinying, Li Zhi Population Migration Algorithm In proteins FOLDING Chongqing:! UNIVERSITY (NATURAL SCIENCE: EDITION) 2007, 3 (2): 110-113

Jian-Wei Ma, Guo-Li Zhang Application of artificial fish-swarm neural network it short-term load forecasting of power system .. Power System Technology, Jun 5, 2005: 36-39

MY, Jiang; Y, Wang; Pfletschinger, S, et al .. Optimal multiuser detection with artificial fish swarm algorithm Advanced intelligent computing theories and applications, 2007 (2) 1084-1093..

Cui-ru Wang, Chun-lei Zhou, Jian-Wei Ma AN Improved artificial Fish-swarm algorithm and ITS application in feed-forward neural networks International Conference on Machine Learning and Cybernetics, ICMLC 2005: 2890-2894

Guang-qiu, Huang; Qiu-qin, Lu; Guan, Liu An approach to air leakage points identification in branches of ventilation system based on fish-swarm algorithm Journal of System Simulationpournal of System Simulation, 2007:. 2677-2682.

Shelokar, PS; Siarry, P; Jayaraman, VK, et al Particle swarm and ant colony algorithms hybridized for improved continuous optimization.Applied mathematics and compution 2007,188 (1): 129$142 .$. 